IJMMS 26:10 (2001) 615-624

PII. S0161171201004148

http://ijmms.hindawi.com

(c) Hindawi Publishing Corp.

\title{
SOME CONDITIONS ON DOUGLAS ALGEBRAS THAT IMPLY THE INVARIANCE OF THE MINIMAL ENVELOPE MAP
}

\author{
CARROLL GUILLORY
}

(Received 20 November 1999)

\begin{abstract}
We give general conditions on certain families of Douglas algebras that imply that the minimal envelope of the given algebra is the algebra itself. We also prove that the minimal envelope of the intersection of two Douglas algebras is the intersection of their minimal envelope.
\end{abstract}

2000 Mathematics Subject Classification. 46Jxx, 30Dxx.

1. Introduction. Let $D$ denote the open unit disk in the complex plane, and $T$ the unit circle. By $L^{\infty}$ we mean the space of essential bounded measurable functions on $T$ with respect to the normalized Lebesgue measurement. We denote by $H^{\infty}$ the space of all bounded analytic functions in $D$. Via identification with boundary functions, $H^{\infty}$ can be considered as a uniformly closed subalgebra of $L^{\infty}$. Any uniformly closed subalgebra $B$ strictly between $H^{\infty}$ and $L^{\infty}$ is called a Douglas algebra $B$. If $C$ is the set of all continuous functions on $T$, we set $H^{\infty}+C=\left\{h+g: h \in H^{\infty}, g \in C\right\}$. Then $H^{\infty}+C$ becomes the smallest Douglas algebra containing $H^{\infty}$ properly.

The function

$$
q(z)=\prod_{n=1}^{\infty} \frac{\left|z_{n}\right|}{z_{n}} \frac{z-z_{n}}{1-\bar{z}_{n} z}
$$

is called a Blaschke product if $\sum_{n=1}^{\infty}\left(1-\left|z_{n}\right|\right)$ converges. The set $\left\{z_{n}\right\}$ is called the zero set of $q$ in $D$. Here $\left|z_{n}\right| / z_{n}=1$ is understood whenever $z_{n}=0$. We call $q$ an interpolating Blaschke product if

$$
\inf _{n} \prod_{m: m \neq n}\left|\frac{z_{m}-z_{n}}{1-\bar{z}_{n} z_{m}}\right|>0
$$

An interpolating Blaschke product $q$ is called sparse (or thin) if

$$
\lim _{n \rightarrow \infty} \prod_{m: m \neq n}\left|\frac{z_{m}-z_{n}}{1-\bar{z}_{n} z_{m}}\right|=1 \text {. }
$$

The set $Z(q)=\left\{x \in M\left(H^{\infty}\right) \backslash D: q(x)=0\right\}$ is called the zero set of $q$ in $M\left(H^{\infty}+C\right)$. Any function $h$ in $H^{\infty}$ with $|h|=1$ a.e. on $T$ is called an inner function. Since $|q|=1$ for any Blaschke product, Blaschke products are inner functions. Let $Q C=\left(H^{\infty}+C\right) \cap$ $\overline{H^{\infty} \cap C}$ and for $x \in M\left(H^{\infty}+C\right)$, set

$$
Q_{x}=\left\{y \in M\left(L^{\infty}\right): f(x)=f(y) \forall f \in Q C\right\}
$$


Then $Q_{x}$ is called the $Q C$-level set for $x$. For $x \in M\left(H^{\infty}+C\right)$, we denote by $\mu_{x}$ the representing measure for $x$, and its support set by supp $\mu_{x}$. By $H^{\infty}[\bar{q}]$ we mean the Douglas algebra generated by $H^{\infty}$ and the complex conjugate of the function $q$. Since $M\left(L^{\infty}\right)$ is the Shilov boundary for every Douglas algebra, a closed set $E$ contained in $M\left(L^{\infty}\right)$ is called a peak set for a Douglas algebra $B$ if there is a function $f$ in $B$ with $f=1$ on $E$ and $|f|<1$ on $M\left(L^{\infty}\right) \backslash E$. A closed set $E$ is a weak peak set for $B$ if $E$ is the intersection of a family of peak sets. If the set $E$ is a weak peak set for $H^{\infty}$ and we define

$$
H_{E}^{\infty}=\left\{f \in L^{\infty}:\left.\left.f\right|_{E} \in H^{\infty}\right|_{E}\right\},
$$

then $H_{E}^{\infty}$ is a Douglas algebra. For a Douglas algebra $B$, we define $B_{E}$ similarly.

For an interpolating Blaschke product $q$ we put $N(\bar{q})$ the closure of

$$
\cup\left\{\operatorname{supp} \mu_{x}: x \in M\left(H^{\infty}+C\right),|q(x)|<1\right\} .
$$

Then $N(\bar{q})$ is a weak peak set for $H^{\infty}$. By $N_{0}(\bar{q})$ we denote the closure of $\cup\left\{\operatorname{supp} \mu_{x}\right.$ : $x \in Z(q)\}$. In general $N_{0}(\bar{q})$ does not equal $N(\bar{q})$, but in this paper $N_{0}(\bar{q})=N(\bar{q})$. For $x \in M\left(H^{\infty}\right)$, we let $E_{x}=\left\{y \in M\left(H^{\infty}\right)\right.$ : supp $\left.\mu_{y}=\operatorname{supp} \mu_{x}\right\}$ and call $E_{x}$ the level set of $x$. Since the sets supp $\mu_{x}$ and $N(\bar{q})$ are weak peak sets for $H^{\infty}$, both $H_{\text {supp } \mu_{x}}^{\infty}$ and $H_{N(\bar{q})}^{\infty}$ are Douglas algebras. For the interpolating Blaschke product $q$, set

$$
A=\bigcap_{x \in M\left(H^{\infty}+C\right)}\left\{H_{\text {supp } \mu_{x}}^{\infty}: q(x)<1\right\}, \quad A_{0}=\bigcap\left\{H_{\text {supp } \mu_{x}}^{\infty}: x \in Z(q)\right\} .
$$

Our assumptions on $q$ throughout imply that $H_{N(\bar{q})}^{\infty}=A=A_{0}$ (see [8]). For $x$ and $y$ in $M\left(H^{\infty}\right)$, the pseudohyperbolic distance $\rho$ is defined by

$$
\rho(x, y)=\operatorname{supp}\left\{|h(y)|:\|h\|_{\infty} \leq 1, h \in H^{\infty}, h(x)=0\right\} .
$$

For $x$ and $y$ in $D$, we have

$$
\rho(x, y)=\left|\frac{x-y}{1-\bar{y} x}\right| .
$$

For $x \in M\left(H^{\infty}\right)$, we define the Gleason part $P_{x}$ of $x$ by

$$
P_{x}=\left\{y \in M\left(H^{\infty}\right): \rho(x, y)<1\right\} .
$$

If $P_{x} \neq\{x\}$, then $x$ is said to be a nontrivial point. Let $G=\left\{x \in M\left(H^{\infty}+C\right): P_{x}\right.$ is a nontrivial part . We say that an interpolating Blaschke product $q$ is of type $G$ if the modules is 1 on every trivial part, that is, $\left\{x \in M\left(H^{\infty}+C\right):|q(x)|<1\right\} \subset G$. An interpolating Blaschke product $q$ of type $G$ is said to be of finite type $G$ if for every $x \in Z(q)$, the set $Z(q) \cap P_{x}$ is a finite set.

A point $x \in Z(q)$, where $q$ is interpolating Blaschke product, is called a minimal element (or a minimal support point) for the Douglas algebra $H^{\infty}[\bar{q}]$ if there are no $y \notin$ $M\left(H^{\infty}[\bar{q}]\right)$ such that $\operatorname{supp} \mu_{y} \subseteq \operatorname{supp} \mu_{x}$; that is, if $y \notin M\left(H^{\infty}[\bar{q}]\right)$ then either supp $\mu_{y} \cap$ $\operatorname{supp} \mu_{x}=\theta$ or $\operatorname{supp} \mu_{x} \subseteq \operatorname{supp} \mu_{y}$. We let $F=\{x \in G: x \in Z(q), q$ is finite type $\}$. A point $x \in M\left(H^{\infty}\right)$ is called locally thin if there is an interpolating Blaschke product $q$ such that $q(x)=0$ and

$$
\left(1-\left|Z_{n(\alpha)}\right|^{2}\right)\left|q^{\prime}\left(Z_{n(\alpha)}\right)\right| \rightarrow 1
$$


whenever $Z_{n(\alpha)}$ is a subnet of the zero sequence $\left\{Z_{n}\right\}$ of $q$ in $D$ converging to $x$. A point $x \in M\left(H^{\infty}\right)$ is called locally thin if there exists a Blaschke product $q$ which is locally thin at $x$. Since for locally thin points $P_{x}$ is nontrivial, $x \in G$. Let $L=$ $\{x \in G: x$ is a locally thin point $\}$, we will show that $F \subset L$. Finally, we let $m_{q}=\{x \in$ $M\left(H^{\infty}+C\right) \mid x$ is a minimal support point of $\left.H^{\infty}[\bar{q}]\right\}$.

Let $B$ a Douglas algebra. The Bourgain algebra $B_{b}$ of $B$ relative to $L^{\infty}$ is the set of $f$ in $L^{\infty}$ such that $\left\|f f_{n}+B\right\|_{\infty} \rightarrow 0$ for every sequence $\left\{f_{n}\right\}$ in $B$ with $f_{n} \rightarrow 0$ weakly in $B$. An algebra $B$ is called a minimal superalgebra of an algebra $A$ if $A \subset B$ and $\operatorname{supp} \mu_{y}=\operatorname{supp} \mu_{x}$ for all $x, y \in M(A) \backslash M(B)$. The minimal envelope $B_{m}$ of a Douglas algebra $B$ is defined to be the smallest Douglas algebra that contains all the minimal superalgebra of $\dot{B}$. The mapping that assigns to $B$ the Douglas algebra $B_{m}$ is called the minimal envelope map and the mapping that assigns to $B$ the Douglas algebra $B_{b}$ is called the Bourgain map.

Let $E_{\text {thin }}=\left[H^{\infty}: \bar{q} \mid q\right.$ is a thin interpolating Blaschke product $], E_{\mathrm{fin}}=\left[H^{\infty}: \bar{b} \mid \boldsymbol{b}\right.$ is of finite type $G]$, and $E_{G}=\left[H^{\infty}: \bar{\varphi} \mid \varphi\right.$ is of type $\left.G\right]$. Then it is clear that $E_{\text {thin }} \subseteq E_{\text {fin }} \subseteq E_{G}$.

2. Some Douglas algebra $B$ with the property $B_{m}=B$. We begin with the following theorems.

THEOREM 2.1. Let $q$ be an interpolating Blaschke product of type $G$. Let $B=H_{N(\bar{q})}^{\infty}$. Then $B_{m}=B$.

Proof. Let $b$ be an interpolating Blaschke product with $\bar{b} \notin B$. Then there is an $m \in M(B)$ such that $b(m)=0$. Since $B=\bigcap_{x \in Z(q)} H_{\operatorname{supp} \mu_{x}}^{\infty}$ then there is a $y \in Z(q)$ such that $\bar{b} \notin H_{\text {supp } \mu_{y}}^{\infty}$. We can assume that $\operatorname{supp} \mu_{m} \subseteq \operatorname{supp} \mu_{y}$. This implies $|b(y)|<1$. Let $\left\{Z_{n}\right\}$ be the zero sequence of $q$ in $D$. Since $Z(q)=\left\{\bar{Z}_{n}\right\} \backslash\left\{Z_{n}\right\}$ and $y \in Z(q)$, there is a subsequence $\left\{Z_{n \beta}\right\}$ such that $Z_{n \beta} \rightarrow y$ as $\beta \rightarrow \infty$. Since $\left|b\left(Z_{n \beta}\right)\right| \rightarrow|b(y)|$ as $\beta \rightarrow \infty$, there is a positive integer $N$ and an $\epsilon$ with $0<\epsilon<1$ such that $\left|b\left(Z_{n \beta}\right)\right|<1-\epsilon$ for all

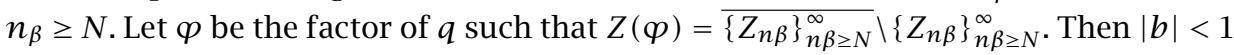
on $Z(\varphi)$. By [12, Theorem 1], there are uncountable infinitely many $x, y \in Z(\varphi)$ such that $\operatorname{supp} \mu_{x} \cap \operatorname{supp} \mu_{y}=\theta$. This implies that there are infinitely many $x, y \in Z(\varphi)$ such that $E_{x} \cap E_{y}=\theta$ and $\bigcup_{x \in Z(\varphi)} E_{x} \subset\{m \in M(B):|b(m)|<1\}$. By [9, Theorem 3] we have that $b \notin B_{m}$. This proves our theorem.

THEOREM 2.2. $\left[E_{\mathrm{fin}}\right]_{m}=E_{\mathrm{fin}}$.

Proof. Let $q$ be any interpolating Blaschke product such that $\bar{q} \notin E_{\mathrm{fin}}$. Then $q$ is not of finite type $G$ (nor the product of a finite number of finite type $G$ ). Hence by [10, Theorem 3.1], there are $x$ and $y$ in $M\left(H^{\infty}+C\right)$ such that $|q(x)|<1,|q(y)|<1$ and $\operatorname{supp} \mu_{x} \subseteq \operatorname{supp} \mu_{y}$.

CASE 1. Suppose $y \in M\left(E_{\text {fin }}\right)$. Then by [8, Theorem 2] there is an uncountable infinite index set $I$ such that (a) for each $\alpha \in I$, there is an $x_{\alpha} \in M\left(H^{\infty}+C\right)$ with $\operatorname{supp} \mu_{x_{\alpha}} \subseteq \operatorname{supp} \mu_{y}$; (b) $x_{\alpha} \in Z(q)$; and (c) for $\alpha, \beta \in I, \alpha \neq \beta$, supp $\mu_{x_{\alpha}} \cap \operatorname{supp} \mu_{x_{\beta}}=\theta$. Since $y \in M\left(E_{\text {fin }}\right)$ we have that $x_{\alpha} \in M\left(E_{\text {fin }}\right)$ for all $\alpha \in I$. Thus $\bigcup_{\alpha \in I} E_{x_{\alpha}} \subset\{m \in$ $\left.M\left(E_{\text {fin }}\right) \mid q(m)<1\right\}$. By [9, Theorem 3], $q \notin\left[E_{\text {fin }}\right]_{m}$.

CASE 2. If $y \notin M\left(E_{\mathrm{fin}}\right)$. Then again for each $\alpha \in I$, we have that $x_{\alpha} \in M\left(E_{\mathrm{fin}}\right)$. This follows from [11, Theorem 9] which states that every invertible interpolating Blaschke 
product in $E_{\text {fin }}$ is the product of a finite number of interpolating Blaschke product of finite type $G$. So if $b$ is invertible in $E_{\text {fin }}$, and $|b(y)|<1$, then $\left|b\left(x_{\alpha}\right)\right|=1$ for all $\alpha \notin I$ by [9, Theorem 3.1]. Thus $x_{\alpha} \in M\left(E_{\text {fin }}\right)$ for all $\alpha \in I$, and so $\bigcup_{\alpha \in I} E_{x_{\alpha}} \subset\left\{m \in M\left(E_{\text {fin }}\right) \mid\right.$ $|q(y)|<1\}$. This implies that $\bar{q} \notin\left[E_{\mathrm{fin}}\right]_{m}$. Therefore $E_{\mathrm{fin}}=\left[E_{\mathrm{fin}}\right]_{m}$.

THEOREM 2.3. $E_{\text {thin }}=\left[E_{\text {thin }}\right]_{m}$.

Proof. Let $\varphi$ be an interpolating Blaschke product such that $\bar{\varphi} \notin\left[E_{\text {thin }}\right]$. Since $E_{\text {thin }} \subseteq E_{\text {fin }}$, we have that $\left[E_{\text {thin }}\right]_{m} \subseteq\left[E_{\text {fin }}\right]_{m}=E_{\text {fin }}$. We can assume that $\bar{\varphi} \in E_{\text {fin. }}$. We will show that there is an infinite set $F \subseteq Z(\varphi)$ such that for any $x, m \in F$, supp $\mu_{x} \cap$ $\operatorname{supp} \mu_{m}=\theta$ and $\bigcup_{x \in F} \subset\left\{w \in M\left(E_{\text {thin }}\right)|| \varphi(w) \mid<1\right\}$. By [9, Theorem 3.1] this will show that $\bar{\varphi} \notin\left[E_{\text {thin }}\right]_{m}$. Hence will show that $\left[E_{\text {thin }}\right]_{m}=E_{\text {thin }}$. Since $\bar{\varphi} \notin E_{\text {thin }}$ there is an $x_{0} \in M\left(E_{\text {thin }}\right)$ such that $\varphi\left(x_{0}\right)=0$. Let $\left\{Z_{n}\right\}$ be the zero sequence of $\varphi$ in $\dot{D}$. Since $\varphi$ is interpolating and $Z(\varphi)=\left\{\bar{Z}_{n}\right\} \backslash\left\{Z_{n}\right\}$, there exists a subsequence $\left\{Z_{n \beta}\right\}$ such that $Z_{n \beta} \rightarrow x_{0}$. We will show that the set $\left\{\bar{Z}_{n B}\right\} \backslash\left\{Z_{n h}\right\} \subset M\left(E_{\text {thin }}\right)$. Let $q$ be any interpolating Blachke product such that $\bar{q} \in E_{\text {thin }}$ (then $q$ is the product of a finite number of thin interpolating Blaschke product). Since $x_{0} \in M\left(E_{\text {thin }}\right)$, we have that $\left|q\left(x_{0}\right)\right|=1$. Thus $\left|q\left(Z_{n \beta}\right)\right| \rightarrow 1$ as $\beta \rightarrow \infty$. This shows that $|q|=1$ on $\left\{\bar{Z}_{n \beta}\right\} \backslash\left\{Z_{n \beta}\right\}$. Since this is true for any $q$ with $\bar{q} \in E_{\text {thin }}$, we have

$$
\left\{\bar{Z}_{n \beta}\right\} \backslash\left\{Z_{n \beta}\right\} \subset M\left(E_{\text {thin }}\right) .
$$

Since $\left\{\bar{Z}_{n \beta}\right\} \backslash\left\{Z_{n \beta}\right\}$ is homeomorphic to the compactification of the integer, we have that $\left\{\bar{Z}_{n \beta}\right\} \backslash\left\{Z_{n \beta}\right\}$ is uncountably infinite and by [12, Theorem 1], there are infinite many $x, y \in\left\{\bar{Z}_{n \beta}\right\} \backslash\left\{Z_{n \beta}\right\}$ such that $\operatorname{supp} \mu \cap \operatorname{supp} \mu_{y}=\theta$. Thus if $F=\left\{\bar{Z}_{n \beta}\right\} \backslash\left\{Z_{n \beta}\right\}$, then we have

$$
\bigcup_{m \in F} E_{m} \subset\left\{w \in M\left(E_{\text {thin }}\right) \mid \varphi(w)<1\right\} .
$$

Hence $\bar{\varphi} \notin\left[E_{\text {thin }}\right]_{m}$. This proves our theorem.

We are unable to give solutions to the following two problems.

Problem 2.4. Let $q$ be an interpolating Blaschke product that is not of type $G$. Is it true that $\left(H_{N(\bar{q})}^{\infty}\right)_{m}=H_{N(\bar{q})}^{\infty}$ ?

Let $B$ be any Douglas algebra, $B \neq H^{\infty}+C$, and let $W$ be a weak peak set for $B$. Mortini and Younis have shown that the restricted algebra $H_{W}^{\infty}$ has the property that $\left[H_{W}^{\infty}\right]_{m}=\left[H_{W}^{\infty}\right]_{b}=H_{W}^{\infty}$, if $W$ is a weak peak set for $H^{\infty}$.

Problem 2.5. What conditions must be imposed on the Douglas algebra $B$ such that $B_{W}=\left[B_{W}\right]_{m}$ ? For example, if $B=H^{\infty}[\bar{q}]$, where $q$ is an interpolating Blaschke product of type $G$, and $W$ is a weak peak set for $B$, then is $B_{W}=\left[B_{W}\right]_{m}$ ?

Problem 2.6. Is $\left[E_{G}\right]_{m}=E_{G}$ ?

We also have the following theorem. The case for the Bourgain algebra $B_{b}$ has been proven in [15]. The proof used here is quite different from theirs, and can be used to show that his result also holds for the Bourgain algebras.

THEOREM 2.7. Let $A$ and $B$ be Douglas algebras. Then $(A \cap B)_{m}=A_{m} \cap B_{m}$. 
Proof. Since $A \cap B$ is contained in both $A$ and $B$ by [9, Proposition 6], $(A \cap B)_{m}$ is contained in both $A_{m}$ and $B_{m}$. Hence $(A \cap B)_{m} \subset A_{m} \cap B_{m}$.

To show that $A_{m} \cap B_{m} \subset(A \cap B)_{m}$, let $\varphi$ be an interpolating Blaschke product such that $\bar{\varphi} \in A_{m} \cap B_{m}$. We show that $\bar{\varphi} \in(A \cap B)_{m}$. By [9, Theorem D] we can assume that there is an $x \in M(A)$ and a $y \in M(B)$ such that $\{\lambda \in M(A):|\psi(\gamma)|<1\}=E_{x}$ and $\{\mu \in M(B):|\psi(\mu)|<1\}=E_{y}$. Thus

$$
\begin{aligned}
M(A \cap B) & =M(A) \cup M(B)=M(A[\bar{\psi}] \cap B[\bar{\psi}]) \cup E_{x} \cup E_{y} \\
& =M((A \cap B)[\bar{\psi}]) \cup E_{x} \cup E_{y} .
\end{aligned}
$$

Hence $\left\{w \in(A \cap B)_{m}:|\psi(w)|<1\right\}=E_{x} \cup E_{y}$. By [9, Theorem 3] we have $\bar{\psi} \in(A \cap B)_{m}$. Then our theorem follows.

The following corollaries are immediate consequences of the theorem.

COROLlary 2.8. Let $A$ and $B$ be Douglas algebras with $A=A_{m}$ and $B=B_{m}$. Then $A \cap B=(A \cap B)_{m}$.

COROLLARY 2.9. Let $B_{0}$ be a Douglas algebra with $\left(B_{0}\right)_{m}=B_{0}$ and $B$ be a Douglas algebra such that there is an interpolating Blaschke product $q$ with $M\left(B_{0}\right) \cap$ $M\left(H^{\infty}[\bar{q}]\right)=M(B) \cap M\left(H^{\infty}[\bar{q}]\right)$. If $A$ is a Douglas algebra with the property $A_{m}=A$, then $(B[\bar{\varphi}] \cap A)_{m}=B[\bar{q}] \cap A$. In particular, $H^{\infty}[\bar{\varphi}] \cap A=\left(H^{\infty}[\bar{\varphi}] \cap A\right)_{m}$ for every interpolating Blaschke product $\varphi$.

Proof. Our hypothesis implies that $B_{0}[\bar{q}]=B[\bar{q}]$, hence $[B[\bar{q}]]_{m}=B[\bar{q}]$. So $B[\bar{q}] \cap$ $A=(B[\bar{q}] \cap A)_{m}$ follows from our theorem. By [2, Theorem 1] we have $H_{m}^{\infty}=H^{\infty}+C$, so by our theorem $H_{m}^{\infty}[\bar{q}] \cap A=\left[H^{\infty}+C\right][\bar{q}] \cap A$.

THEOREM 2.10. Let $B_{0}$ be a Douglas algebra such that $\left(B_{0}\right)_{m}=B_{0}$. Let $B$ be any other Douglas algebra such that there is an interpolating Blaschke product $\varphi$ with $M\left(B_{0}\right) \cap M\left(H^{\infty}[\bar{\varphi}]\right)=M(B) \cap M\left(H^{\infty}[\bar{\varphi}]\right)$. For an interpolating Blaschke product $q$ set $B_{x}=B[\bar{\varphi}] \cap H_{\text {supp } \mu_{x}}^{\infty}$ for each $x \in Z(q)$. Put $B_{e}=\cap\left\{B_{x}: x \in Z(q)\right\}$. Then if $q$ is of type $G$, then $\left(B_{e}\right)_{m}=B_{e}$.

Proof. We show that $B_{e}=B[\bar{\varphi}] \cap H_{N(\bar{q})}^{\infty}$. By an unpublished result of D. Sarason,

$$
\begin{aligned}
M\left(B_{e}\right) & =M\left(\bigcap_{x \in Z(q)} B_{x}\right)=\overline{\bigcup_{x \in Z(q)} M\left(B_{x}\right)}=\overline{\bigcup_{x \in Z(q)} M\left(B[\bar{\varphi}] \cap H_{\left.\operatorname{supp} \mu_{x}\right)}^{\infty}\right.} \\
& =\overline{\bigcup_{x \in Z(q)}\left[M ( B [ \overline { \varphi } ] ) \cap M \left(H_{\left.\left.\operatorname{supp} \mu_{x}\right)\right]}^{\infty}\right.\right.}=M(B[\bar{\varphi}]) \cup \overline{\bigcup_{x \in Z(q)} M H_{\operatorname{supp} \mu_{x}}^{\infty}}
\end{aligned}
$$

Now, if $q$ is of type $G$, [7, Proposition 1] and [10, Theorem 3.2(i)] implies $\bigcap_{x \in Z(q)} H_{\text {supp } \mu_{x}}^{\infty}=H_{N(\bar{q})}^{\infty}$. Thus

$$
\overline{\bigcup_{x \in Z(q)} M\left(H_{\text {supp } \mu_{x}}^{\infty}\right)}=M\left(H_{N(\bar{q})}^{\infty}\right)
$$


and we get

$$
M\left(B_{e}\right)=M(B[\bar{\varphi}]) \cup M\left(H_{N(\bar{q})}^{\infty}\right)=M\left(B[\bar{\varphi}] \cap H_{N(\bar{q})}^{\infty}\right) .
$$

So by the Chang-Marshall theorem $[1,13]$ we have $B_{e}=B[\bar{\varphi}] \cap H_{N(\bar{q})}^{\infty}$.

The condition $M\left(B_{0}\right) \cap M\left(H^{\infty}[\bar{\varphi}]\right)=M(B) \cap M\left(H^{\infty}[\bar{\varphi}]\right)$, implies that $B[\bar{\varphi}]=B_{0}[\bar{\varphi}]$. Hence $[B[\bar{\varphi}]]_{m}=\left[B_{0}[\bar{\varphi}]\right]_{m}=\left[B_{0}\right]_{m}[\bar{\varphi}]=B_{0}[\bar{\varphi}]=B[\bar{\varphi}]$, where the middle equality follows from [9, Theorem 4]. By Theorem 2.1 (and its proof) we have $\left(H_{N(\bar{q})}^{\infty}\right)_{m}=H_{N(\bar{q})}^{\infty}$ if $\bar{q}$ is of type $G$. Thus

$$
\left(B_{e}\right)_{m}=\left(B[\bar{\varphi}] \cap H_{N(\bar{q})}^{\infty}\right)_{m}=B[\bar{\varphi}] \cap H_{N(\bar{q})}^{\infty}=B_{e} .
$$

This proves our theorem.

THEOREM 2.11. Let $\left\{q_{n}\right\}$ be a sequence of interpolating Blaschke product that are not invertible in a Douglas algebra A. Suppose for each $n$ there is a Douglas algebra $A_{n}$ with $\left[A_{n}\right]_{m}=A_{n}$ and $M(A) \cap M\left(H^{\infty}\left[\bar{q}_{n}\right]\right)=M\left(A_{n}\right) \cap M\left(H^{\infty}\left[\bar{q}_{n}\right]\right)$. Let $B=\bigcap_{n=1}^{\infty} A\left[\bar{q}_{n}\right]$. Then $B_{m}=B$.

Proof. Since $B \subseteq A\left[\bar{q}_{n}\right]$ for $n=1,2, \ldots$, by [9, Proposition 6] we have $B_{m} \subseteq\left(A\left[\bar{q}_{n}\right]\right)_{m}$ for all $n$. Since $M(A) \cap M\left(H^{\infty}[\bar{q}]\right)=M\left(A_{n}\right) \cap M\left(H^{\infty}[\bar{q}]\right)$, we have $A\left[\bar{q}_{n}\right]=A_{n}\left[\bar{q}_{n}\right]$. So using [9, Theorem 4] we have

$$
\left[A\left[\bar{q}_{n}\right]\right]_{m}=\left[A_{n}\left[\bar{q}_{n}\right]\right]_{m}=\left[A_{n}\right]_{m}\left[\bar{q}_{n}\right]=A_{n}\left[\bar{q}_{n}\right]=A\left[\bar{q}_{n}\right] .
$$

Thus $B_{m} \subseteq \bigcap_{n=1}^{\infty}\left[A\left[\bar{q}_{n}\right]\right]_{m}=\bigcap_{n=1}^{\infty} A\left[\bar{q}_{n}\right]=B$.

COROLLARY 2.12. If $B=\bigcap_{n=1}^{\infty} H^{\infty}\left[\bar{q}_{n}\right]$, then $B_{m}=B$.

Before we prove our next theorem, we have the following related lemmas. Lemma 2.13 shows that the set $F \subset L$.

LEMMA 2.13. Let $q$ be an interpolating Blaschke product that is of finite type $G$. Then each $x \in Z(q)$ is a locally thin point. That is $F \subset L$.

Proof. Since $q$ is of finite type $G$, the set $Z(q) \cap P_{x}$ is a finite set, hence there is a factor $q_{0}$ of $q$ such that $Z(q) \cap P_{x}=\{x\}$. Hence $H_{\text {supp } \mu_{x}}^{\infty}\left[\bar{q}_{0}\right]$ is a minimal superalgebra of $H_{\text {supp } \mu_{x}}^{\infty}$. Hence $H_{\text {supp } \mu_{x}}^{\infty} \subset H_{\text {supp } \mu_{x}}^{\infty}\left[\bar{q}_{0}\right]$. By [14, Theorem 5] we have that $x$ is a locally thin point.

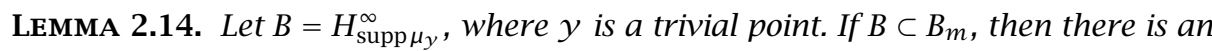
interpolating Blaschke product $q$ and an $x \in Z(q)$ such that $\operatorname{supp} \mu_{x}=\operatorname{supp} \mu_{y}$.

PROoF. If $B \subset B_{m}$ then, by [8, Theorem D], there is an interpolating Blaschke product $q$ such that $B[\bar{q}]$ is a minimal superalgebra of $B$. Hence supp $\mu_{y}$ is a minimal support set of $H^{\infty}[\bar{q}]$. By [6, Theorem 2] there is an $x \in Z(q)$ such that $\operatorname{supp} \mu_{x}=\operatorname{supp} \mu_{y}$.

THEOREM 2.15. Let $q$ be an interpolating Blaschke product and set $\tilde{m}_{q}=\left\{y \in m_{q}\right.$ : $\operatorname{supp} \mu_{y}=\operatorname{supp} \mu_{t}, t$ is a trivial point $\}$. Set $T=\bigcap_{y \in \tilde{m}_{q}} H_{\operatorname{supp} \mu_{y}}^{\infty}$. Then $T_{b}=T$.

Proof. Suppose $T \neq T_{b}$. Then by [9, Theorem $C$ ] there is an interpolating Blaschke product $C$ such that $Z(C) \cap M(T)=\{y\}$. By [7, Theorem 1] we have $M(T)=M(T[\bar{C}]) \cup$ 
$P_{y}$ and $y$ is a minimal support point of $H^{\infty}[\bar{C}]$. By [14, Proposition 6], $\left(H_{\text {supp } \mu_{y}}^{\infty}\right)_{b}=$ $H_{\text {supp } \mu_{y}}^{\infty}[\bar{C}]$. So by [14, Theorem 5], $y$ is a locally thin point. We show that this is not the case by showing that there is $x \in \tilde{m}_{q}$ with $\operatorname{supp} \mu_{x}=\operatorname{supp} \mu_{y}$. By the remark following the proof of [14, Theorem 5], this will lead to a contradiction. Suppose that $\operatorname{supp} \mu_{y} \neq \operatorname{supp} \mu_{x}$ for all $x \in \tilde{m}_{q}$. Then for each $x \in \tilde{m}_{q}$ one of the following must occur. (i) supp $\mu_{x} \subset \operatorname{supp} \mu_{y}$ (properly contained in); (ii) $\operatorname{supp} \mu_{x} \cap \operatorname{supp} \mu_{y}=\theta$; or (iii) $\operatorname{supp} \mu_{y} \subset \operatorname{supp} \mu_{x}$ (properly contained in). We show that none of these can happen.

Suppose (i) is true. Then $|C(x)|=1$ which implies that $\bar{C} \in H_{\operatorname{supp} \mu_{x}}^{\infty}$, since $y$ is a minimal support point of $C$. Thus (i) cannot hold for any $x \in \tilde{m}_{q}$. If (ii) holds for all $x \in \tilde{m}_{q}$, then again $\bar{C} \in T$. Hence neither (i) or (ii) can hold for all $x \in \tilde{m}_{q}$.

Now if (iii) holds for some $x \in \tilde{m}_{q}$, then by [8, Theorem 2] there is an uncountable set $\Gamma \subset Z(C)$ such that supp $\mu_{\alpha} \subseteq \operatorname{supp} \mu_{x}$ (properly contained in) for all $\alpha \in \Gamma$ and $\operatorname{supp} \mu_{\alpha} \cap \operatorname{supp} \mu_{\beta}=\theta$ for all $\alpha, \beta \in \Gamma$ and $\alpha \neq \beta$. Since $x \in M(T)$ we have $\alpha \in M(T)$, hence $\Gamma \subset M(T) \cap Z(C)$. This implies that $Z(C) \cap M(T) \neq\{y\}$. This leads to a contradiction. Thus (iii) cannot hold for any $x \in \tilde{m}_{q}$. So if there is a $y \in M(T) \cap Z(C)$ such that $T[\bar{C}]$ is a minimal superalgebra for $T$, then there is a $x \in \tilde{m}_{q}$ with $\operatorname{supp} \mu_{y}=\operatorname{supp} \mu_{x}$. This implies that $x$ is a locally thin point, which is impossible. Thus $T_{b}=T$.

Is it true that $F=L$ ? That is, every locally thin point is the zero of an interpolating Blaschke product of finite type $G$.

Problem 2.16. What if $F$ is properly contained in $L$. Suppose $x \in L \backslash F$. Is it true that $\operatorname{supp} \mu_{x}$ is maximal?

Both $B_{b}$ and $\mathrm{B}_{m}$ are generated by a special type of minimal superalgebras (determined by the character of the minimal support point).

Under certain conditions we can determine the Bourgain algebra and the minimal envelope algebras of maximal subalgebras of a Douglas algebra $B$ (here $B$ will have a maximal subalgebra).

THEOREM 2.17. Let $B$ be a Douglas algebra such that $B_{b}[\bar{q}]=B[\bar{q}]$ or $B_{m}[\bar{q}]=B[\bar{q}]$ for some interpolating Blaschke product q. Let $A_{x}$ be the maximal subalgebra of $B[\bar{q}]$ for which $x$ is the corresponding minimal support point of $B[\bar{q}]$. Then either

(i) $A_{x} \subset\left(A_{x}\right)_{b}$ and $\left(A_{x}\right)_{b}=\left(A_{x}\right)_{m}=B[\bar{q}]$, or

(ii) $A_{x}=\left(A_{x}\right)_{b}$ and $\left(A_{x}\right)_{m}=B[\bar{q}]$.

Proof. By [14, Theorem 3], [5, Theorem 3], and [9, Theorem 4], our hypothesis implies that $B[\bar{q}]=(B[\bar{q}])_{b}=(B[\bar{q}])_{m}$.

Now let $A_{x}$ be any maximal subalgebra of $B[\bar{q}]$ associated with the minimal support point $x$. First we assume that $x$ is a locally thin point (see [14, Theorem 5]). Then the maximal ideal space of $B[\bar{q}]$ and $A_{x}$ are related by the equation

$$
M\left(A_{x}\right)=M(B[\bar{q}]) \cup P_{x}
$$

Then $B[\bar{q}]$ is a minimal superalgebra of $A_{x}$, hence $B[\bar{q}] \subset\left(A_{x}\right)_{b}$. Now using [14, Theorem 3] again, we get

$$
B[\bar{q}]=(B[\bar{q}])_{b}=\left(A_{x}[\bar{q}]\right)_{b}=\left(A_{x}\right)_{b}[\bar{q}]=\left(A_{x}\right)_{b} \quad \text { since } \bar{q} \in\left(A_{x}\right)_{b} .
$$


Similarly $B[\bar{q}]=\left(A_{x}\right)_{m}=\left(A_{x}\right)_{b}$ if $x$ is a locally thin point. This proves (i).

Now suppose that $x$ is not a locally thin point (e.g., $P_{x}$ is not a homeomorphic disk, see [3]). Then $M\left(A_{x}\right)$ and $M(B[\bar{q}])$ are related by

$$
M\left(A_{x}\right)=M(B[\bar{q}]) \cup E_{x}
$$

with $P_{x} \subseteq E_{x}$ (or $Z(q) \cap P_{x}$ has infinitely many points). Using [9, Theorem 4] we have

$$
\begin{aligned}
B[\bar{q}] & =(B[\bar{q}])_{m}=\left(A_{x}[\bar{q}]\right)_{m} \\
& =\left(A_{x}\right)_{m}[\bar{q}] \quad \text { by }[6, \text { Theorem 4] } \\
& =\left(A_{x}\right)_{m} \quad \text { since } \bar{q} \in\left(A_{x}\right)_{m} .
\end{aligned}
$$

Now, for any Douglas algebra $A$ we have that $A \subseteq A_{b} \subseteq A_{m}$. Thus

$$
A_{x} \subseteq\left(A_{x}\right)_{b} \subseteq B[\bar{q}]=\left(A_{x}\right)_{m} .
$$

Since $A_{x}$ is a maximal subalgebra of $B[\bar{q}]$, we have that $A_{x}=\left(A_{x}\right)_{b}$ if $x$ is not locally thin. This proves (ii).

COROLLARY 2.18. Let $q$ be any interpolating Blaschke product and set $B=H^{\infty}[\bar{q}]$. Let $A_{x}$ be any maximal subalgebra of $B$ that corresponds to the minimal support point of $B$. Then either

(i) $A_{x} \subset\left(A_{x}\right)_{b}$ and $\left(A_{x}\right)_{b}=\left(A_{x}\right)_{m}=B$, or

(ii) $A_{x}=\left(A_{x}\right)_{b}$ and $\left(A_{x}\right)_{m}=B$.

COROLLARY 2.19. Let $A$ be any interpolating Douglas algebra such that $A=A_{b}$ or $A=A_{m}$, and let $q$ be any interpolating Blaschke product such that $\bar{q} \notin A$. Set $B=A[\bar{q}]$ and let $B_{x}$ be any maximal subalgebra of $B$ corresponding to the minimal support point of $A[\bar{q}]$. Then either

(i) $B_{x} \subset\left(B_{x}\right)_{b}$ and $\left(B_{x}\right)_{b}=\left(B_{x}\right)_{m}=B$, or

(ii) $B_{x}=\left(B_{x}\right)_{b}$ and $\left(B_{x}\right)_{m}=B$.

THEOREM 2.20. Let $B$ be a Douglas algebra that has a maximal subalgebra $A_{x}$, where $x$ is the minimal support point of $B$ corresponding to $A_{x}$. Then $\left(A_{x}\right)=B_{m}$.

Proof. By [9, Theorem 4] we have that $\left(A_{x}\right)_{m} \subseteq B_{m}$ since $A_{x} \subseteq B$. Since $A_{x}$ is a maximal subalgebra of $B$ there is an $x_{0} \in M(A) \backslash M(B)$ and an interpolating Blaschke product $\psi_{0}$ such that

$$
M\left(A_{x}\right)=M\left(A\left[\bar{\psi}_{0}\right]\right) \cup E_{x_{0}}=M(B) \cup E_{x_{0}} .
$$

So by [9, Theorem D] we have $B \subseteq\left(A_{x}\right)_{m}$. If $B_{0}$ is another superalgebra containing $A_{x}$, then there is some $y_{0} \in M\left(A_{x}\right)$ such that $M\left(A_{x}\right)=M\left(B_{0}\right) \cup E_{y_{0}}$. Hence we have that $B_{0} \subseteq\left(A_{x}\right)_{m}$ and $y_{0} \in M(B)$, otherwise $E_{y_{0}}=E_{x_{0}}$. To show that $B_{m} \subseteq\left(A_{x}\right)_{m}$, let $\psi$ be any interpolating Blaschke product such that $\bar{\psi} \in B_{m}$. Then by [9, Theorem D] we can assume that

$$
\{\lambda \in M(B):|\psi(\lambda)|<1\}=E_{x}
$$


for some $x \in M(B)$. But

$$
\left\{m \in M\left(A_{x}\right): \psi(m)<1\right\}=E_{x} \cup\left\{m \in M\left(A_{x}\right):|\psi(m)|<1\right\} \cap E_{x_{0}} .
$$

The set on the right-hand side is either $E_{x}$ or $E_{x} \cup E_{x_{0}}$. Hence by [9, Theorem 4] we have that $\bar{\psi} \in\left(A_{m}\right)_{m}$.

For $\left(A_{x}\right)_{b}$ we have the following special result if we assume an additional assumption.

THEOREM 2.21. Let $B$ be a Douglas algebra that has a maximal subalgebra $A_{x}$, where $x$ is the minimal support point of $B$ corresponding to $A_{x}$. Assume that $P_{x}$ is a nonhomeomorphic disk. Then $\left(A_{x}\right)_{b} \subseteq B_{b}$.

Proof. Since $B \subseteq B_{b}$ and $\left(A_{x}\right)_{b} \subseteq B_{b}$, it suffices to show that $B \subsetneq\left(A_{x}\right)_{b}$. Since $A_{x}$ is a minimal subalgebra of $B$ corresponding to $x$, by [7, Theorem 1] we have

$$
M\left(A_{x}\right)=M(B) \cup E_{x}
$$

Note that $P_{x} \subset E_{x}$. Hence if $\psi$ is any interpolating Blaschke product with $\bar{\psi} \in B$, but $\bar{\psi} \notin A_{x}$. Then we have, by [5, Corollary 1.5], the set $M\left(A_{x}\right) \cap Z(\psi) \supseteq P_{x} \cap Z(\psi)$ is an infinite set. Then $\bar{\psi} \notin\left(A_{x}\right)_{b}$. Hence $B \nsubseteq\left(A_{x}\right)_{b}$. (See [4, Theorem 2].)

The following two propositions on minimal support points seem to indicate that the sets given in them are smaller in some sense than the set in the following two well-known facts.

FACT 1. Let $B$ be any Douglas algebra. Then an interpolating Blaschke product $q$ is invertible in $B$ if and only if $Z(q) \cap M(B)=\theta$.

FACT 2. For any Douglas algebra $B$ we have

$$
B=\bigcap_{x \in M(B)} H_{\text {supp } \mu_{x}}^{\infty}
$$

Proposition 2.22. An interpolating Blaschke product $q$ is invertible in a Douglas algebra $B$ if and only if $M(B) \cap m_{q}=\theta$.

Proof. We have that $m_{q} \subseteq Z(q)$, hence if $m_{q} \cap M(B) \neq \theta$ then $\bar{q} \notin B$. (See [7, Theorem 2].)

To prove the converse, suppose that $\bar{q} \notin B$. Then by Fact $1, Z(q) \cap M(B) \neq \theta$. There is a $y_{0} \in Z(q)$ such that $\operatorname{supp} \mu_{y_{0}} \subseteq \operatorname{supp} \mu_{x}$ for any $x \in Z(q) \cap M(B)$ and $y_{0} \in m_{q}$. (See [7, Theorem 2].) Since $x \in M(B)$ we have that $M\left(H_{\operatorname{supp} \mu_{x}}^{\infty} \subset M(B)\right.$. Since $M\left(H_{\operatorname{supp} \mu_{x}}^{\infty} \subset\right.$ $M\left(L^{\infty}\right) \cup\left\{\lambda \in M\left(H^{\infty}+C\right): \operatorname{supp} \mu_{\lambda} \subseteq \operatorname{supp} \mu_{x}\right\}$, we have that $y_{0} \in M(B)$. Hence $m_{q} \cap$ $M(B) \neq \theta$.

Let $B$ be any Douglas algebra and set $m_{q}(B)=m_{q} \cap M(B)$. Let $M_{B}=\cup\left\{m_{q}(B)\right.$ : $q$ is an interpolating Blaschke product, $\bar{q} \notin B\}$. Let $\Gamma(B)=\left\{x_{\alpha}\right\}_{\alpha \in \Lambda}$ be the family of all minimal support points for $M_{B}$ such that $\operatorname{supp} \mu_{\alpha} \cap \operatorname{supp} \mu_{\beta}=\theta$ if $\alpha \neq \beta$. Then

Proposition 2.23. $B=\bigcap_{x_{\alpha} \in \Gamma(B)} H_{\text {supp } \mu_{\alpha}}^{\infty}$. 
Proof. If $x \in M_{B}$, then there is an $x_{\alpha} \in \Gamma(B)$ such that $\operatorname{supp} \mu_{x_{\alpha}}=\operatorname{supp} \mu_{x}$. So it suffices to show that

$$
B=\bigcap_{x \in M_{B}} H_{\operatorname{supp} \mu_{x}}^{\infty}
$$

Set $B_{0}=\bigcap_{x \in M_{B}} H_{\text {supp } \mu_{x}}$. Since $M_{B} \subseteq M(B)$, by Fact 2 we have that $B \subseteq B_{0}$. Suppose $B \subseteq B_{0}$. Then by the Chang-Marshall theorem $[1,13]$ there is an interpolating Blaschke product $q$ such that $\bar{q} \in B_{0}$ but $\bar{q} \notin B$. Hence there is a $y \in(B)$ such that $q(y)=0$. Hence $\bar{q} \notin H_{\text {supp } \mu_{y_{0}}}$, so $\bar{q}$ cannot be invertible in $B_{0}$. Thus $B_{0}=B$.

ACKNOWLEDGEMENT. All work on this paper was done while the author was at the Mathematical Science Research Institute. The author thanks the Institute for its support during this period. Research at MSRI is supported in part by NSF grant DMS9022140.

\section{REFERENCES}

[1] S. Y. A. Chang, A characterization of Douglas subalgebras, Acta Math. 137 (1976), no. 2, 81-89. MR 55\#1074a. Zbl 332.46035.

[2] J. A. Cima, S. Janson, and K. Yale, Completely continuous Hankel operators on $H^{\infty}$ and Bourgain algebras, Proc. Amer. Math. Soc. 105 (1989), no. 1, 121-125. MR 89g:30065. Zbl 709.30032.

[3] T. W. Gamelin, Uniform Algebras, Chelsea Publishing, New York, 1984.

[4] P. Gorkin, K. Izuchi, and R. Mortini, Bourgain algebras of Douglas algebras, Canad. J. Math. 44 (1992), no. 4, 797-804. MR 94c:46104. Zbl 763.46046.

[5] P. Gorkin, H.-M. Lingenberg, and R. Mortini, Homeomorphic disks in the spectrum of $H^{\infty}$, Indiana Univ. Math. J. 39 (1990), no. 4, 961-983. MR 92b:46082. Zbl 799.46061.

[6] C. Guillory, Douglas algebras that have no maximal subalgebras and no minimal subalgebras, MSRI preprint series 1995-083.

[7] C. Guillory and K. Izuchi, Maximal Douglas subalgebras and minimal support points, Proc. Amer. Math. Soc. 116 (1992), no. 2, 477-481. MR 92m:46075. Zbl 760.46046.

[8]_ Interpolating Blaschke products and nonanalytic sets, Complex Variables Theory Appl. 23 (1993), no. 3-4, 163-175. MR 95c:46076. Zbl 795.30031.

[9]__ Minimal envelopes of Douglas algebras and Bourgain algebras, Houston J. Math. 19 (1993), no. 2, 201-222. MR 94i:46067. Zbl 816.46048.

[10] _ Interpolating Blaschke products of type G, Complex Variables Theory Appl. 31 (1996), no. 1, 51-64. MR 97i:46090. Zbl 865.30054.

[11] C. Guillory and K. Y. Li, Algebras of Blaschke products unimodular on trivial parts, Complex Variables Theory Appl. 35 (1998), no. 4, 307-318. MR 99e:46064. Zbl 893.30024.

[12] K. Izuchi, QC-level sets and quotients of Douglas algebras, J. Funct. Anal. 65 (1986), no. 3, 293-308. MR 87f:46093. Zbl 579.46037.

[13] D. E. Marshall, Subalgebras of $L^{\infty}$ containing $H^{\infty}$, Acta Math. 137 (1976), no. 2, 91-98. MR 55\#1074b. Zbl 334.46061.

[14] R. Mortini and R. Younis, Douglas algebras which are invariant under the Bourgain map, Arch. Math. (Basel) 59 (1992), no. 4, 371-378. MR 94c:46105. Zbl 760.46050.

[15] R. Younis and D. Zheng, A distance formula and Bourgain algebras, Math. Proc. Cambridge Philos. Soc. 120 (1996), no. 4, 631-641. MR 97f:46087. Zbl 878.46042.

CARroll Guillory: Department of MAthematics, University of Louisiana AT LAFAYETTE, LAFAYETTE, LA 70504, USA

E-mail address: cjg2476@1ouisiana.edu 


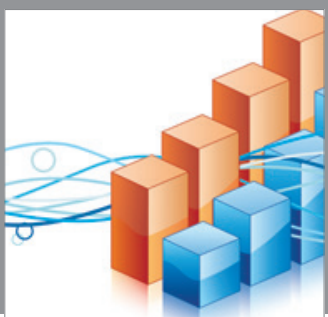

Advances in

Operations Research

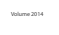

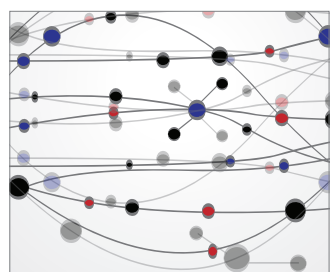

\section{The Scientific} World Journal
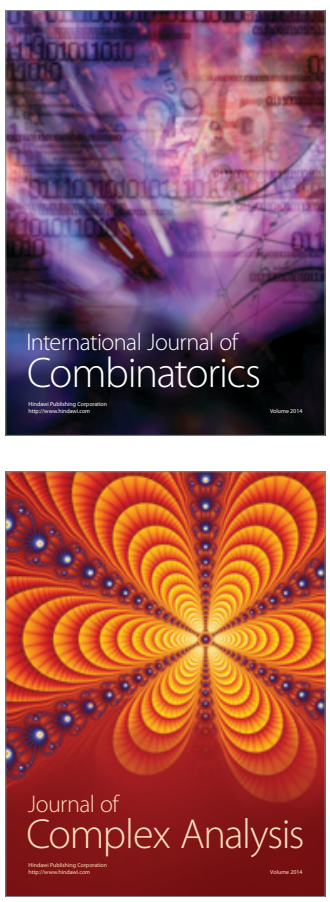

International Journal of

Mathematics and

Mathematical

Sciences
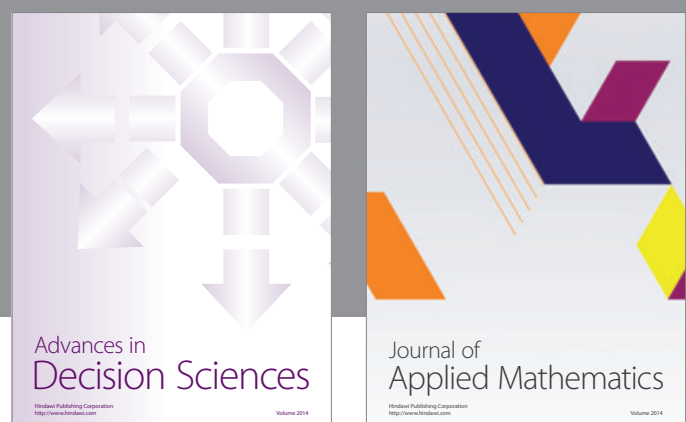

Journal of

Applied Mathematics
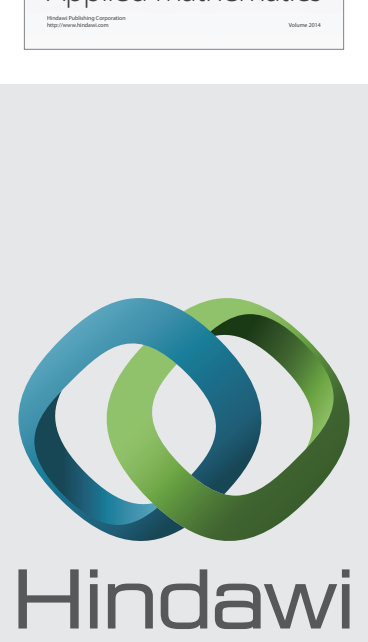

Submit your manuscripts at http://www.hindawi.com
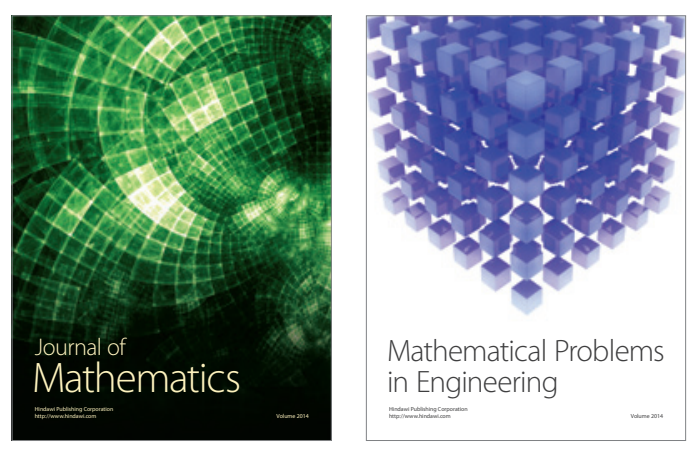

Mathematical Problems in Engineering
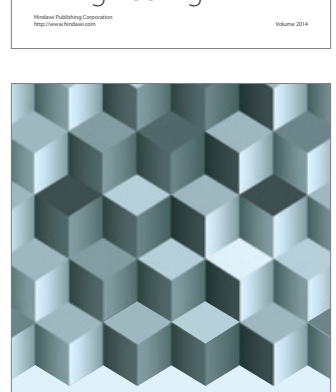

Journal of

Function Spaces
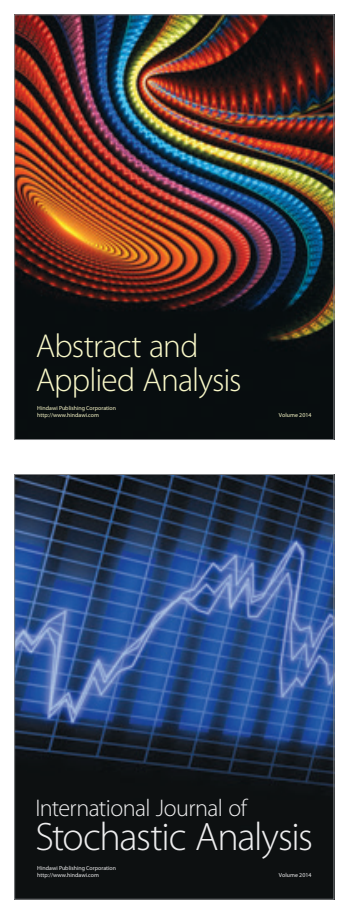

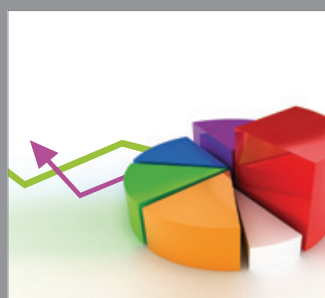

ournal of

Probability and Statistics

Promensencen
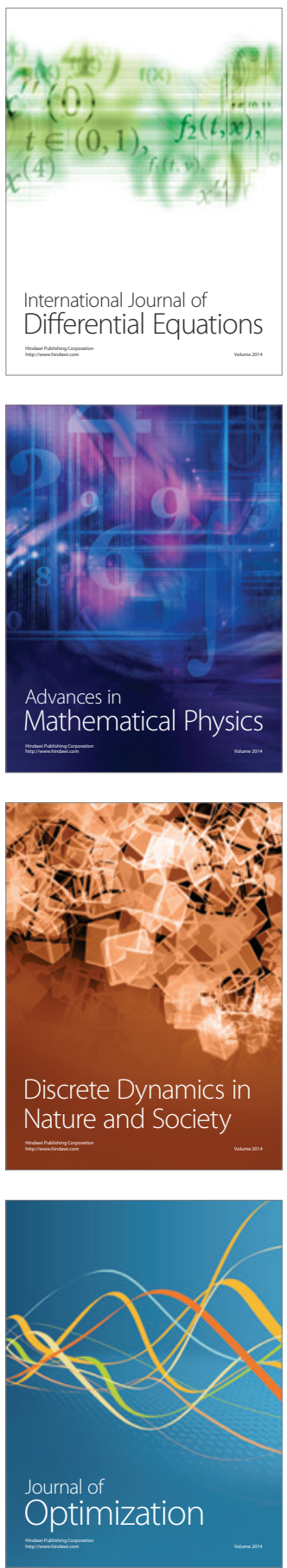